\title{
THE RADIO MORPHOLOGY OF QUASARS AT HIGH REDSHIFT
}

\author{
P. D. Barthel \\ Owens Valley Radio Observatory \\ California Institute of Technology \\ Pasadena, California 91125 \\ USA
}

The mapping at kpc-scale resolution of the radio sources associated with quasars is fascinating, since it provides us with morphological information on a population of objects during $10^{10.3} \mathrm{y}$ evolution of the universe, as well as information on a possible epoch dependence of the influence of the ambient medium on the properties of these objects.

In 1980 we started a project to map all Quasi Stellar Radio Sources (QSRs) having $z>1.5$ and steep or unknown radio spectrum. We extracted the QSRs from the Hewitt \& Burbidge (1980) compilation, and used the spectral window $1.4 \mathrm{GHz}$ (or $408 \mathrm{MHz}$ ) - $5 \mathrm{GHz}$ with the criterion $\alpha \gtrsim 0.6$ for the steep spectrum sources. The sources originated in the $4 \mathrm{C}$, B2, MC, PKS, Ohio, and Westerbork Catalogues, and a considerable fraction had only one measured spectral point. Using the VLA at $5 \mathrm{GHz}$, we obtained angular resolutions of $0.4^{\prime \prime}-0.5^{\prime \prime}$. Although in the following I will only discuss the $5 \mathrm{GHz}$ results, we have in the meantime obtained many complementary data using the VLA at $15 \mathrm{GHz}$, MERLIN at 1.67 and $5 \mathrm{GHz}$, and the European VLBI Network at $1.67 \mathrm{GHz}$ (resolutions between $0.03^{\prime \prime}$ and $\left.0.3^{\prime \prime}\right)$. The $5 \mathrm{GHz}$ VLA observations resulted in radio maps, polarization data and spectral indices for some 100 QSRs, having $z$ between 1.502 and 3.19.

Although the Fanaroff \& Riley (1974) classification would predict edge-brightened double sources for these distant, powerful QSRs, we find a predominance of small, distorted morphologies at high $z$. It is well known that redshift and intrinsic luminosity are correlated in flux density limited samples (from which these QSRs originate). However, we were able to separate the effects of the redshift (epoch) and the luminosity, and the distortion turns out to be a redshift effect. Two examples are shown in Figure 1. We find that jet curvature/distortion frequently occurs, usually in highly polarized, very localized areas. These distortions occur both on subgalactic $(0-10 \mathrm{kpc})$ and larger $(>10 \mathrm{kpc})$ scale. Summarizing, at high $z$ the radio jets experience difficulties in the formation of extended radio sources.

It is a well known fact that radio spectral index and the presence of extended (kpc scale) emission regions, containing aging electrons, are tightly correlated. It is therefore interesting to examine whether the above mentioned high $z$ behaviour is reflected in the spectral index distributions at high redshift. Table 1 shows the numbers of flat spectrum and steep spectrum quasars (nota bene: wide spectral window!) for $z>1.5$ in consecutive redshift bins. In brackets are the numbers of unresolved or slightly resolved steep spectrum 
sources, the so called Steep Spectrum Cores (SSCs). The Hewitt \& Burbidge compilation lists 9 QSRs having $z>3$, whereas the most recent figure is 15 .

From Table 1 we find a decrease in the fractions steep/flat and SSC/flat with increasing redshift. There are two possible explanations for this decrease: 1 ) it is real; and 2) we simply do not detect the very distant QSRs having steep spectra, due to observational selection. There are three arguments supporting the reality of the decrease: 1 ) the average value of the spectral index for the SSCs decreases with $z$, indicating evolution within the class of SSCs; 2) although we may miss some of the extended sources at high redshift due to limited surface brightness sensitivity, SSCs at redshifts $4-5$ should be easily detectable, if they were there; 3) since several (high z) QSR surveys were carried out at high frequency (small beam facilitates identification) a high fraction of flat spectrum sources is to be expected. However, none of the QSRs at $z>3$ show a prominent increase in radio flux density towards lower frequencies ( $m$-wavelengths), as do the low redshift core dominated sources (e.g., 3C 273, 3C 345). Although the $z>3$ QSRs tend to be bright at $\mathrm{cm}$-wavelength, they do not appear in low frequency catalogues (e.g., 4C), in agreement with the fact that they do not show extended ( 1 " scale) structure. This third argument can also be used against the hypothesis of the $z>3$ QSRs being beamed towards us: where is the larger scale, unbeamed emission?

We conclude that a decrease in the space density of steep spectrum QSRs at high redshift is very likely.

Apart from the apparent general QSO cutoff (see Osmer's contribution in these Proceedings), there are also two arguments for a flat spectrum QSR cutoff at $z \sim 4$ : 1) all the $z>3$ (flat spectrum) QSRs would be easily detectable at $z=4-5$. They are not found, and dust obscuration does not work; 2) Downes et al. (Edinburgh preprint $15 / 85$ ) found a high identification rate for sources in a complete sample of deep PKS selected regions, implying that the weak sources in this sample, although distant, cannot be extremely distant.

Taking these facts together, we propose that the evolution of the quasar phenomenon from an apparent cutoff at $z \sim 4$, to $z \sim 2$ goes together with a decrease in the density of an initially very dense, presumed galactic, surrounding medium. These dense high $z$ environments initially block the propagation of radio jets, and hence prohibit the formation of extended emission regions.

The properties of these high $z$ environments will be spectroscopically investigated in 1986, using the Palomar 200-inch telescope. Speculations on a physical link between these dense high $z$ environments and the general QSO redshift cutoff are left to the reader.

I wish to thank my collaborators George Miley, Richard Schilizzi and Colin Lonsdale. Full account of this work will be given elsewhere. Until further notice I offer a free beer for every new QSR having $z>2.5$ and a steep $(\mathrm{cm}-\mathrm{m})$ radio spectrum. 
Table 1. The QSR spectral index distributions in bins of increasing redshift.

\begin{tabular}{lllll}
\hline spectral index & $1.5<z<2.0$ & $2.0<z<2.5$ & $2.5<z<3.0$ & $z>3.0$ \\
\hline steep (SSC) & $53(11)$ & $22(5)$ & $6(2)$ & 0 \\
flat & 59 & 38 & 13 & $9(15)$ \\
\hline
\end{tabular}
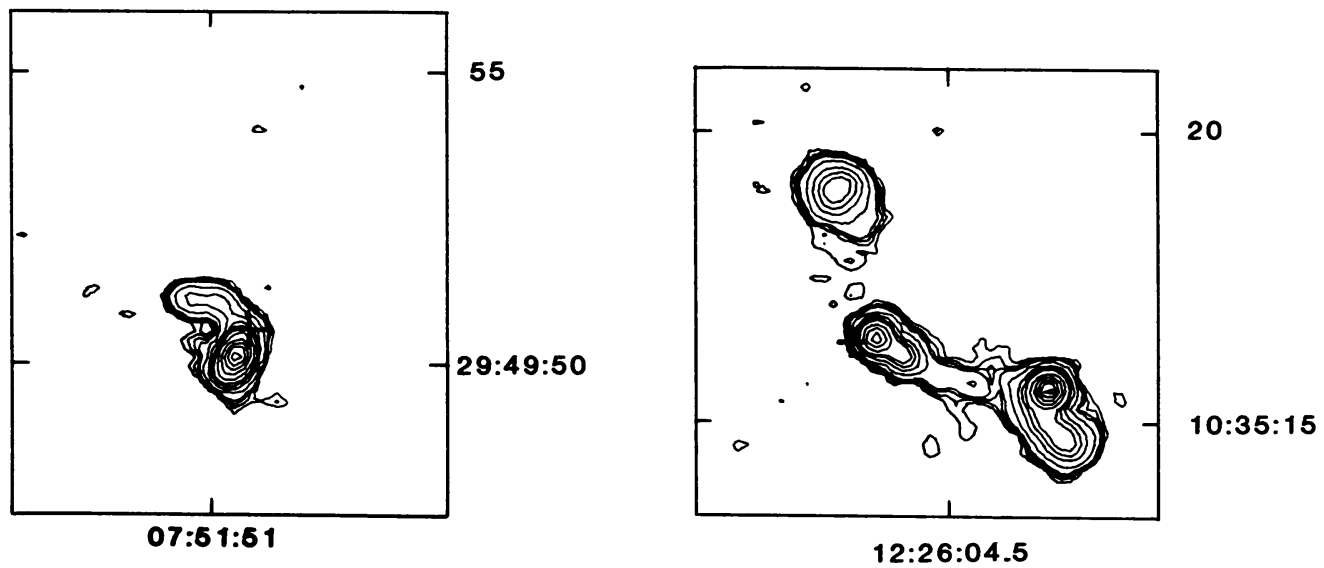

Figure 1. The radio sources associated with the quasars $0751+298(z=2.106)$ and $1226+105(z=2.296)$.

\section{DISCUSSION}

Filippenko : Many of your maps show a substantial offset between the radio core and the optical nucleus. What is your interpretation of this?

Barthel : The scale of the maps, having overall sizes of $5 "$ - $10^{\prime \prime}$ is such that the offsets are not larger than $0 ! 5$ - $0 ! 7$, which is the error in the optical position.

Burke : (1) How dense must the confining galactic medium be in the high-z quasar case ? (2) Is the lack of coincidence between the optical quasar position and a visible radio core caused by uncertainity in the astrometry ?

Barthel : (1) $10^{2}-10^{4} \mathrm{~cm}^{-3}$. (2) Yes.

Murdoch : Many QSOs have steep spectrum at low frequency and flat spectrum at high frequency. Hence for a given observed frequency range, the same source might be observed with a steep spectrum if it occurs at low 
redshift or with a flat spectrum if it occurs at high redshift due to the large ratio of intrinsic to observed frequency. This could explain at least partially the preponderance of flat spectrum sources at high redshift.

Barthel : I agree. However, none of the $z>3$ QSRs show a steep increase in flux density towards low frequency (m-wavelength), in agreement with their generally unresolved morphology. On the other hand, Savage \& Peterson (Early Evolution of the Universe, IAU Symp., ed. Abell \& Chincarini, p. 57) draw attention to the reverse effect.

Kapahi : The lack of very high $z$ quasars in the low frequency surveys could be understood due to the very steep radio luminosity function. If flat spectrum quasars are similar to the normal double quasars, differing only in orientation, one would indeed expect flat spectrum-quasars to extend to higher redshifts because of relativistic beaming.

Barthel : The effect may be important to some extent. My main comment is that we should see a steep increase in flux density towards $m$-wavelengths, due to the unbeamed component(s), as is the case for e.g. 3C345. This is not seen for the QSRs at $z>3$.

Kuhr : In your sample you did not find extended radio structure for quasars with $z>3$; but there exists at least one quasar of redshift $z=3.41$, the most luminous quasar, for which that was found with the VLA at an angular distance of $0 ! 6$ from the nucleus.

Barthel : I was fully aware of that, but considered the overall extent of the source not impressive. Apart from that, also the spectrum of this quasar is not steep. However, the extended component may be the radio jet, finding its way out.

Wilkes : I understood from Fraser Owen's talk that confining and bending of extended structure in low-redshift QSOs cannot be explained in terms of the surrounding medium. Is there any reason why a high density gas should be doing the confining at high-redshift.

Barthel : Frazer Owen was referring to static, thermal confinement of radio plasma in a hot ICM. The cases I showed were subgalactic hotspots with minimum internal pressures $10^{-6}-10^{-5}$ dyne $\mathrm{cm}^{-2}$. Ram pressure confinement by a very dense (clumpy?) galactic medium seems the only plausible mechanism.

McAdam : Your abstract mentions violent interaction of the radio jets with a medium. What are the indications of violence and do you believe the medium is within or surrounding any galactic (10 kpc) scale?

Barthel : Calculating the momentum transfer onto a deflecting/confining medium one finds values of about $10^{2} \mathrm{M}_{0} \times 10^{3} \mathrm{~km} \mathrm{~s}{ }^{-1}$ per year, which I consider violent. In these cases the size scales are 5-10 kpc from the nucleus $\left(H_{0}=75, q_{0}=1 / 2\right)$. 